\title{
The Method for Placement of an Intraoperative Continuous Facial Nerve Stimulating Electrode in Acoustic Neuroma Surgery: Technical Note
}

\author{
Koichi ToriHaSHI, ${ }^{1,2}$ Shigeo SORA, ${ }^{1}$ Hiroaki SATO, ${ }^{1}$ and Michihiro KOHNO ${ }^{1,3}$ \\ ${ }^{1}$ Department of Neurosurgery and Stroke Center, \\ Tokyo Metropolitan Police Hospital, Tokyo, Japan; \\ ${ }^{2}$ Division of Neurosurgery, Department of Brain and Neurosciences, \\ Faculty of Medicine, Tottori University, Yonago, Tottori, Japan; \\ ${ }^{3}$ Department of Neurosurgery, Tokyo Medical University Hospital, Tokyo, Japan
}

\begin{abstract}
Preservation of facial nerve (FN) function is the most important goal in acoustic neuroma (AN) surgery. We have been using intraoperative continuous facial nerve monitoring (ICFNm) of evoked electromyography during AN surgery. ICFNm is very useful, and we can identify the real-time functions of the FN. Some surgeons have experienced difficulty with placing the ICFN stimulating electrode (SE). We therefore show how to place the ICFN SE. We mostly perform AN surgery with a retrosigmoid approach (RSA). A craniotomy with four burr holes is performed. We dissect the arachnoid membrane along the accessory nerve from the cisterna magna to the glossopharyngeal nerve. When we are able to identify the root exit zone (REZ) of the FN near the brainstem, we place the ICFN SE on it. However, when a large tumor covers the REZ, we have to debulk the tumor to create a space between the tumor and the glossopharyngeal nerve. After that, we can place the SE on the REZ. A method for placement of the ICFN SE is needed for some techniques of AN surgery. Once we learn how to place the SE, we can identify continuous FN function during AN surgery. This method is useful for the preservation of postoperative FN function in AN surgery.
\end{abstract}

Key words: acoustic neuroma, vestibular schwannoma, facial nerve monitoring, retrosigmoid approach

\section{Introduction}

Preservation of facial nerve (FN) function is one of the primary purposes of acoustic neuroma (AN) surgery. Surgical techniques and equipment have been described to preserve FN function. ${ }^{1-6)}$ Because postoperative FN dysfunction impairs the patient's quality of life, the main purpose of AN surgery is maximal tumor resection with complete functional preservation of the $\mathrm{FN}^{1-6)}$ We have been using intraoperative continuous monitoring of evoked FN electromyography (EMG) in addition to conventional methods. ${ }^{4,7)}$ This technique involves continuous electrical stimulation with a stimulating electrode (SE) that is placed on the root exit zone (REZ). This method is useful for identification of changes in FN

Received June 13, 2018; Accepted August 2, 2018

Copyright $\odot 2018$ by The Japan Neurosurgical Society This work is licensed under a Creative Commons AttributionNonCommercial-NoDerivatives International License.
EMG during removal of the tumor, and to confirm potential problems in real time, as described previously. ${ }^{7)}$ Real-time monitoring of latency and amplitude is possible during intraoperative continuous facial nerve monitoring (ICFNm). However, some surgeons have experienced difficulty with placement of the ICFN SE. Therefore, we introduce how to place the ICFN SE with the retrosigmoid approach (RSA) for AN surgery.

\section{Surgical Technique}

Intraoperative FN monitoring is routinely performed with EMG of the frontalis, orbicularis oculi, and orbicularis oris muscles. Three types of monitoring are performed: free-running spontaneous EMG and occasional and continuous evoked FN EMG with direct electrical stimulation..$^{4,7-10)}$ In this ICFNm, the REZ of the FN is electrically stimulated at a frequency of $1 \mathrm{~Hz}$, and the evoked compound muscle action potential of facial muscles is continuously monitored.7) When we identify the REZ around the 
brainstem, we stimulate it and confirm the response of the FN EMG. Then, we place the ICFN SE on the REZ. The ICFN SE is a ball-type monopolar electrode (Ematsu, Tokyo, Japan) $)^{7)}$ (Figs. 1A and 1B).

\section{Skin incision and craniotomy}

The anatomical landmarks are drawn on the skin, including the mastoid, digastric groove, and asterion. The skin incision is C-shaped on the post-auricular region. A craniotomy is performed with four burr holes, exposing the transverse sinus (TS), sigmoid sinus (SS), and their junction. We place the keyhole on the TS-SS junction, defining a single point on the three-dimensional computed tomography (CT) image on the venous phase. The craniotomy is extended inferiorly to the posterior condylar emissary vein. When the tumor is large, sometimes we have to open the foramen magnum.

\section{Dissecting the arachnoid membrane from the cisterna magna to the lateral cerebellomedullary cistern}

U-shaped dural incision is made on the bottom of the SS. The dura is reflected anteriorly. The marginal dura near the edge of the SS is pulled up superiorly, and then we can access the cerebellopontine angle easily with minimal cerebellar retraction.

We open the cisterna magna to aspirate the cerebrospinal fluid to allow slackening of the cerebellum. After that, we dissect the arachnoid along the accessory nerve from the cisterna magna to the lateral cerebellomedullary cistern. We open the lateral cerebellomedullary cistern to dissect the arachnoid around the lower cranial nerve to identify the REZ of the FN.

\section{Placement of the ICFN SE}

We expose the glossopharyngeal nerve from the brainstem to the lateral side and identify the REZ around the brainstem. Sometimes, a large tumor covers the REZ. When identification of the REZ is difficult due to a large tumor, we have to debulk the tumor to create space between the glossopharyngeal nerve and the tumor. We stimulate the dorsal side of the tumor with the probe before debulking the tumor because the FN is occasionally on the dorsal side of the tumor. ${ }^{9)}$ After we rule out a dorsal FN course, we can reduce the tumor size safely. After the tumor size is decreased because of debulking, the space between the glossopharyngeal nerve and the tumor appears or is widened. Then, we can easily identify the REZ around the brainstem (Fig. 2A).

After confirmation of the FN response (the frontalis, orbicularis oculi, and orbicularis oris muscles), when stimulating the REZ with the FN stimulating probe $(0.2-0.4 \mathrm{~mA})$, we place the ICFN SE and fix it using surgicel cotton balls. Frequent measurement of M-max to calculate the amplitude preservation ratio is important in continuous stimulation monitoring. M-max is measured just after placing the ICFN SE, as previously described. ${ }^{7}$ After that, we can remove the tumor safely by placement of the ICFN SE. The schema of the placement of the ICFN $\mathrm{SE}$ is shown in Fig. 2B.

\section{Discussion}

Many types of intraoperative FN monitoring systems have been reported. ${ }^{11-14)}$ Occasional electrical stimulation of the FN using a probe-type SE is a common method in AN surgery. Using this method, we can identify the location and running course of the FN. However, the disadvantage of this monitoring method without continuous stimulation is the inability to identify damage to the $\mathrm{FN}$ in real time during removal of the tumor. Therefore, ICFNm by placing the SE on the REZ is a useful method for preserving
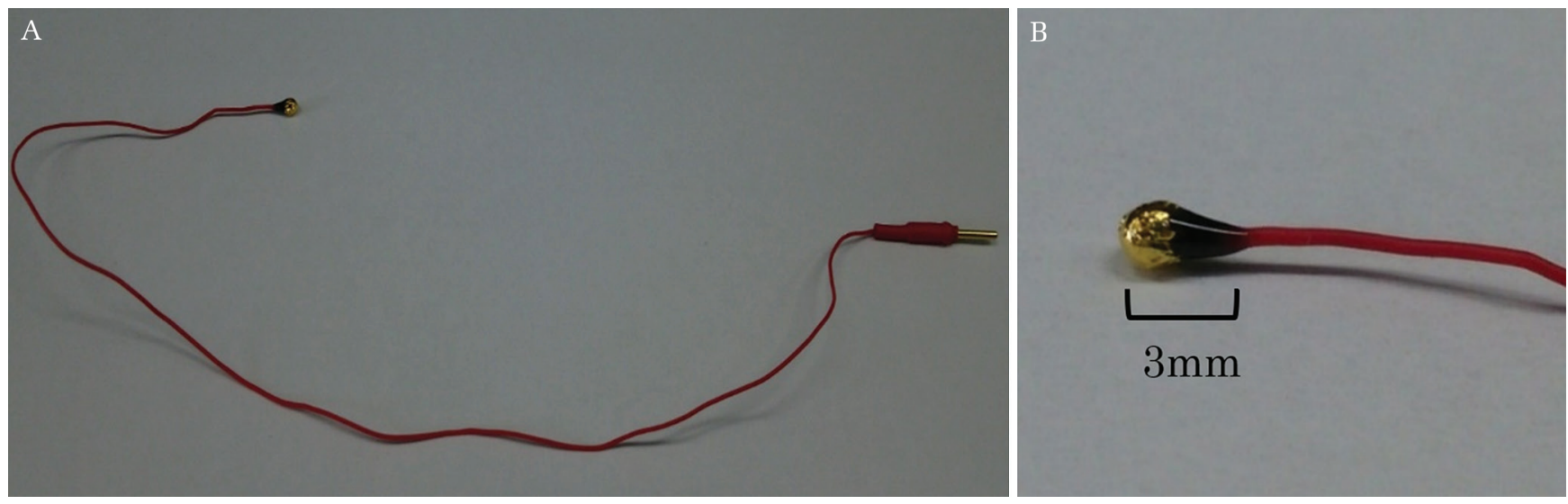

Fig. 1 (A) This is the intraoperative continuous facial nerve stimulating electrode. (B) The ball-type electrode is placed directly on the REZ of the facial nerve. 

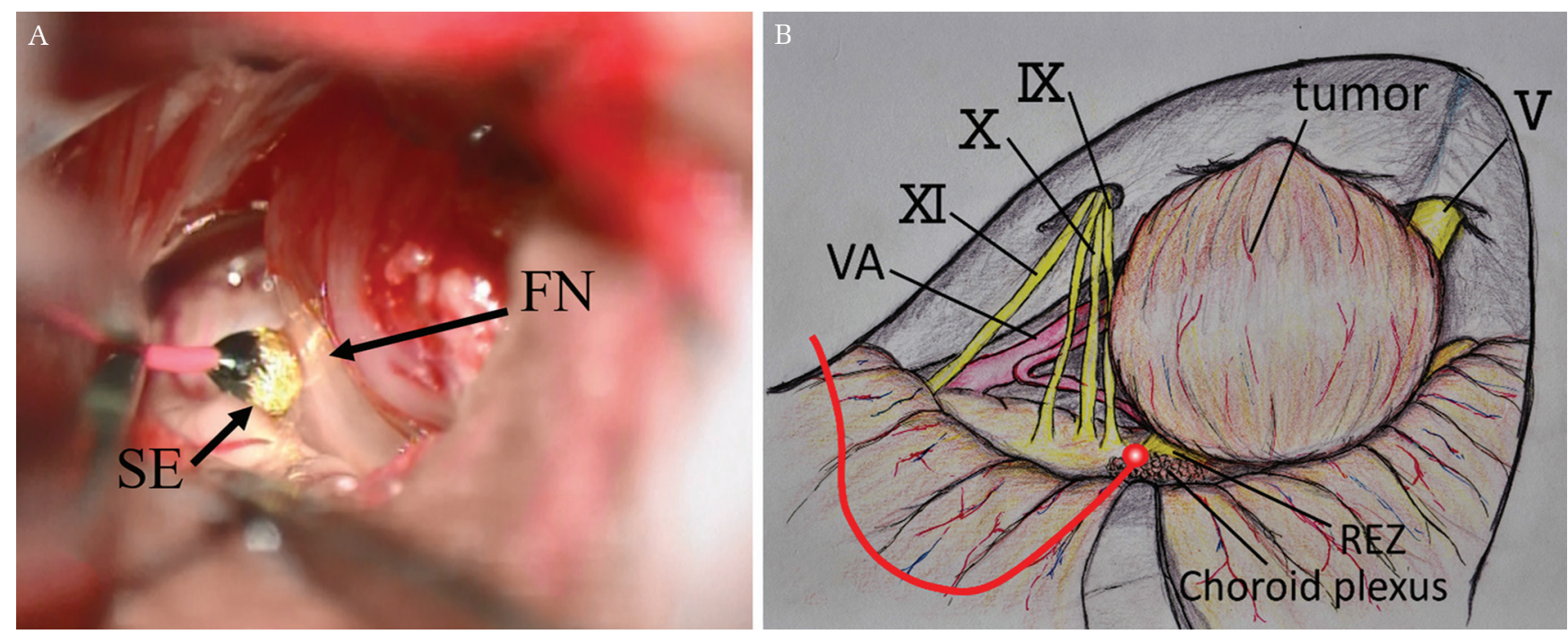

Fig. 2 (A) After identification of the REZ, we stimulate it with probe. After identification of the facial nerve (FN) response, we place the intraoperative continuous facial nerve stimulating electrode (SE) (arrow) on the REZ of FN (arrow). (B) The schema of how to place the electrode on the REZ around FN.

postoperative FN function. ${ }^{4,7)}$ To avoid postoperative facial nerve palsy, a warning criterion of amplitude preservation ratio $>50 \%$ appears useful. ${ }^{7)}$

However, sometimes placing the SE in AN surgery is difficult, because identifying the REZ is difficult in cases in which a large AN covers the REZ. The operative field in AN surgery with the RSA is narrower and deeper than in other neurosurgical operations. However, once we learned how to place the SE, these techniques became more useful. We can monitor the FN function continuously and perform AN surgery safely. We can use this method to place the SE in every AN surgery.

To perform continuous stimulating monitoring effectively, the ICFN SE is fixed on the REZ for constant recording of EMG. However, some problems can develop after placing the SE. When the SE is unstable, electric current on the FN is not constant, resulting in variable wave patterns. The main causes are that the SE may slip and shift during removal of the tumor. One reason for movement of the SE is retraction of the cord of the SE.

Therefore, we need to place the cord of the ICFN SE on the caudal and then lateral side so that it does not obstruct the operative field during tumor removal (Fig. 3). Also, we place a surgicel cotton ball or cottonoid on the SE to stabilize it.

However, sometimes the SE slips during transformation around the REZ due to debulking of the tumor. When the amplitude of EMGs reduces, we should raise the electrical stimulation and confirm the reason.

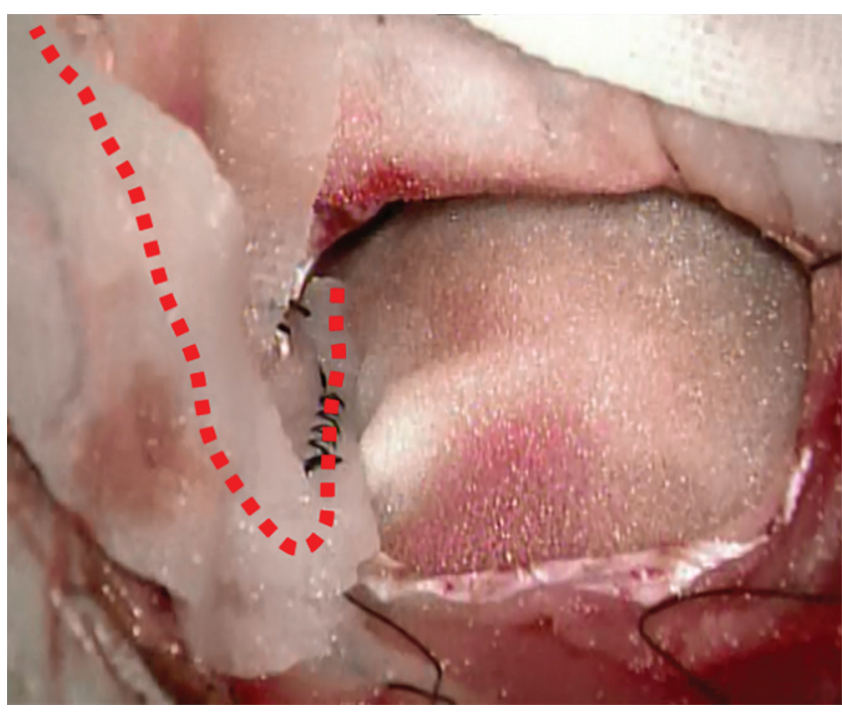

Fig. 3 Red dotted line indicates the cord of the intraoperative continuous facial nerve stimulating electrode.

If the amplitude recovers by raising stimulation, slipping of SE is suspected and we should replace it.

\section{Conclusion}

We reported the techniques for using the ICFN SE during AN surgery with the RSA. A method for placing the SE is needed for some techniques. However, once we learned how to place the SE, we can confirm continuous FN function during AN 
surgery. This method is useful for preservation of postoperative FN function.

\section{Conflicts of Interest Disclosure}

All authors who are a member of the Japan Neurosurgical society (JNS) have registered on line selfreported COI Disclosure Statement Forms through the website. The authors have no interests to declare.

\section{References}

1) Gurgel RK, Dogru S, Amdur RL, Monfared A: Facial nerve outcomes after surgery for large vestibular schwannomas: do surgical approach and extent of resection matter? Neurosurg Focus 33: E16, 2012

2) Kemink JL, Langman AW, Niparko JK, Graham MD: Operative management of acoustic neuromas: the priority of neurologic function over complete resection. Otolaryngol Head Neck Surg 104: 96-99, 1991

3) Koos WT, Day JD, Matula C, Levy DI: Neurotopographic considerations in the microsurgical treatment of small acoustic neurinomas. J Neurosurg 88: 506-512, 1998

4) Kohno M, Taniguchi M: Intraoperative real-time continuous facial nerve monitoring in acoustic neuroma surgery. Acta Neurochir 153: 2273, 2011

5) Nonaka Y, Fukushima T, Watanabe K, et al.: Contemporary surgical management of vestibular schwannomas: analysis of complications and lessons learned over the past decade. Neurosurgery 72: ons103-ons115, 2013

6) Samii M, Matthies C: Management of 1000 vestibular schwannomas (acoustic neuromas): surgical management and results with an emphasis on complications and how to avoid them. Neurosurgery 40: 11-21; discussion 21-23, 1997

7) Amano M, Kohno M, Nagata O, Taniguchi M, Sora $\mathrm{S}$, Sato H: Intraoperative continuous monitoring of evoked facial nerve electromyograms in acoustic neuroma surgery. Acta Neurochir (Wien) 153: 1059-1067, 2011

8) Kohno M, Sato H, Sora S, Miwa H, Yokoyama M: Is an acoustic neuroma an epiarachnoid or subarachnoid tumor? Neurosurgery 68: 1006-1016; discussion 1016-1007, 2011

9) Nejo T, Kohno M, Nagata O, Sora S, Sato H: Dorsal displacement of the facial nerve in acoustic neuroma surgery: clinical features and surgical outcomes of 21 consecutive dorsal pattern cases. Neurosurg Rev 39: 277-288, 2016

10) Kohno M, Shinogami M, Yoneyama H, Nagata $O$, Sora S, Sato H: Prognosis of tinnitus after acoustic neuroma surgery-surgical management of postoperative tinnitus. World Neurosurg 81: 357-367, 2014

11) Tokimura H, Sugata S, Yamahata H, Yunoue S, Hanaya $\mathrm{R}$, Arita K: Intraoperative continuous monitoring of facial motor evoked potentials in acoustic neuroma surgery. Neurosurg Rev 37: 669-676, 2014

12) Dong CC, Macdonald DB, Akagami R, et al.: Intraoperative facial motor evoked potential monitoring with transcranial electrical stimulation during skull base surgery. Clin Neurophysiol 116: 588-596, 2005

13) Grayeli AB, Guindi S, Kalamarides M, et al.: Fourchannel electromyography of the facial nerve in vestibular schwannoma surgery: sensitivity and prognostic value for short-term facial function outcome. Otol Neurol 26: 114-120, 2005

14) Kombos T, Suess O, Kern BC, Funk T, Pietilä T, Brock M: Can continuous intraoperative facial electromyography predict facial nerve function following cerebellopontine angle surgery? Neurol Med Chir (Tokyo) 40: 501-505; discussion 506-507, 2000

Address reprint requests to: Koichi Torihashi, MD, Division of Neurosurgery, Department of Brain and Neurosciences, Faculty of Medicine, Tottori University, 1-36 Nishicho, Yonago, Tottori 683-8504, Japan.

e-mail: ktsep9010@yahoo.co.jp 